\title{
Issues involved in the rapid assessment of the leprosy problem
}

\author{
T K SUNDARESAN
}

Summary Sample surveys for estimation can prove very expensive and timeconsuming because of the enormous sample sizes usually required.

Where sample surveys have to be undertaken, diagnoses should be limited to detecting a case of leprosy, without attempting skin smears etc. in order to classify by types. Usually enough knowledge is available on the approximate proportion of multibacillary (MB) cases in most communities, and this knowledge could be utilized for estimating the caseload by types of leprosy. Again intensive tracing of nonrespondents could be limited to either males or females depending on convenience, and well-known sex ratios among patients utilized for deriving estimates for the other sex.

The type of rapid methods of estimation depend on three types of situations: (1) before multidrug therapy (MDT); (2) 5 years or more after MDT; and (3) less than 5 years after MDT.

In the first situation one or more of the following methods are suggested:

(i) extrapolation from registered cases;

(ii) extrapolation from child prevalence; and

(iii) conducting rapid village surveys.

In situations where MDT has been introduced for 5 years or more the registered cases plus a small number, depending on local experience, would seem to be adequate.

When MDT was introduced less than 5 years before, it is suggested that the prevalence rates be obtained by statistical interpolation drawing on the experience from areas which have had more than 5 years of MDT.

\section{Introduction}

Most of the information now available on the magnitude of the leprosy problem in the world is based on registered cases. Reports from most countries give the numbers actually on the registers and invariably there is comment that the true prevalence of the disease should be at least $50 \%$ or $100 \%$ more. The World Health Organization has underlined the need for reliable baseline information on the prevalence of the disease before introducing MDT. The need for reliable information on the true magnitude of the problem is well understood. This paper will discuss various methods that could possibly be used and highlight the advantages and shortcomings of each such method. 


\section{Estimation and case-finding}

At the outset, a clear distinction needs to be made between case-finding for control purposes and estimation. In case-finding and control, an effort is made to find all possible cases in a community and treat them. On the other hand, the purpose of estimation is generally to get an idea of the caseload in large areas such as a country or a district. The purpose is 2-fold: (1) to obtain baseline information on the magnitude of the problem; and (2) to assess periodically the progress of the control programme. This paper deals only with problems of estimation.

It may be relevant to mention two concepts which should be constantly kept in mind in connection with problems of estimation. The first is 'nonsampling errors'. This can arise due to differences in the definition of a case, observer variations in the diagnosis and classification, incomplete coverage of the population, etc. It is most important to keep these variations to a minimum otherwise the estimates will not be 'valid'. The second consideration is the 'precision' with which the estimates are required. These are the 'sampling errors' and have a direct bearing on the sample size, in the case of sample surveys, and will be discussed more at length in a subsequent section.

\section{Medical issues involved in estimation}

There is not yet a universally accepted definition of a case of leprosy, notwithstanding the recommendation of the WHO Sixth Expert Committee ${ }^{1}$ that introduced a new category'cases needing treatment'. Some investigators would like to count even very minimal lesions with a good prospect of self-healing, while others would like to leave them out. The specificity of diagnosis in early cases is likely to be less than in established cases. Again there are the usual intra- and inter-observer variations in diagnosis of early disease and in the classification of the disease as paucibacillary or multibacillary, depending mostly on the accuracy of bacteriological examinations. It may be easy to categorize patients on the registers according to this criterion, but when enumerators meet cases in surveys, they have to depend on the information of the patients themselves to decide whether they need further treatment or not and this may not be accurate.

\section{Total population surveys}

This procedure should in principle give the exact situation in the community, but it is too expensive, time-consuming and demands a lot of resources in terms of personnel and equipment, and even assuming that resources are available this method is not necessarily the best. The disease is 'rare' in a statistical sense and occurs unevenly or in 'patches'. Nonresponse due to various reasons is all too common. If the nonresponse is related to the occurrence of the disease, as is likely in many situations, this could greatly bias the results. The fact that $99 \%$ of the population has been examined can give a confidence in the findings, but the $1 \%$ not covered could be hiding a disproportionate number of cases. Again there is the question of 'what is a case?' It is well known that a substantial number of paucibacillary cases are self-healing. After all, the main interest is in knowing the numbers needing treatment. Thus a repeat visit may be necessary to exclude the cases that have healed in the meanwhile. For this purpose it is necessary to lay down rules for the periodicity, i.e. at what interval. 
Table 1

\begin{tabular}{lr}
\hline $\begin{array}{l}\text { Expected Prev. rate } \\
\text { (per 1000) }\end{array}$ & Sample size \\
\hline 5 & 230,000 \\
4 & 287,000 \\
3 & 384,000 \\
2 & 576,000 \\
1 & $1,152,000$ \\
\hline
\end{tabular}

\section{SAMPLE SURVEYS}

Since the aim is only to estimate the prevalence of the disease, sample surveys are to be preferred to total enumerations because:

(1) they are cheaper;

(2) the results can be obtained in a shorter time; and

(3) intense effort can be put into controlling the non-sampling errors referred to in the previous section.

However an adequate sample size depends on: (1) the expected prevalence of the disease; and (2) the precision with which the estimates are required. Given the degree of precision required the sample size will increase when the prevalence decreases. Cluster sampling is the usual design of choice, being the most practical, and the design effect is assumed to be about three. (This means that the sample size will be about three times that for simple random sampling.) Table 1 will give an idea of the minimum sample sizes for different 'expected' prevalences for a precision of $20 \%$.

Second, for the same expected prevalence the sample size will increase even more rapidly when the degree of precision demanded increases. Table 2 will give an idea of the order of magnitude of the increases for a prevalence rate of 2 per 1000 .

With the sort of prevalence rates expected in low and moderately endemic areas, the sample sizes have to be very large. Securing improved coverage once the sample has been chosen, and keeping down other nonsampling errors, can be enormous tasks.

\section{'Rapid' methods and suggested simplifications}

Long years of experience in leprosy control in the world have highlighted certain patterns

Table 2

\begin{tabular}{lcrr}
\hline \multirow{2}{*}{$\begin{array}{l}\text { Relative } \\
\text { precision } \\
(\%)\end{array}$} & \multicolumn{2}{c}{$95 \%$ C. limit } & \\
\cline { 2 - 3 } & upper & lower & Sample size \\
\hline 40 & $2 \cdot 4$ & $1 \cdot 6$ & 144,000 \\
30 & $2 \cdot 3$ & $1 \cdot 7$ & 256,000 \\
20 & $2 \cdot 2$ & $1 \cdot 8$ & 576,000 \\
10 & $2 \cdot 1$ & $1 \cdot 9$ & $2,310,000$ \\
\hline
\end{tabular}


in the distribution of the disease in the community and the numbers coming to the knowledge of the leprosy services. These are related to the general attitude of the population and the adequacy of the leprosy services. The epidemiological pattern is seen in the age and sex distribution and the proportion of multibacillary patients among all cases of leprosy. Bechelli et al. $^{2}$ have suggested making use of observations on these patterns to predict the prevalence of the disease.

The introduction of MDT on a large scale is known to affect the patterns referred to above and hence the approach to 'rapid' methods has to visualize three situations:

I The situation before the introduction of MDT on a large scale. Baseline information on the magnitude of the problem is often a prerequisite for introducing MDT and a good part of the endemic areas of the world are in this situation. As MDT is being introduced rapidly there is an urgent need for a method to obtain the information quickly.

II The situation where it is 5 years or more since the introduction of MDT with adequate coverage.

III The situation where it is less than 5 years since MDT has been introduced.

\section{BASELINE INFORMATION BEFORE INTRODUCING MDT}

\section{(i) Sample Surveys}

If there is no information to go by, a sample survey may have to be undertaken. The methodology for such sample surveys in leprosy has been elaborated by Sundaresan et al. ${ }^{3}$ However, the following simplications are suggested:

One of the components of medical examination is the taking of skin smears and testing them bacteriologically. This is an expensive operation and calls for trained personnel. However, from observations of registered cases and limited surveys, the proportion of multibacillary cases seems to be fairly constant in each society although the proportion can vary from country to country. For example, before the introduction of MDT, it was between 16 and $23 \%$ in Nigeria, India and Ethiopia. Bechelli et al. ${ }^{4}$ have reported that in the sample surveys in Cameroon, Northern Nigeria (Katsina) and Thailand (Khon Kaen), in the 1960s, lepromatous and borderline cases constituted $8 \%, 8 \%$ and $37 \%$ of all cases respectively. However, the prevalence of indeterminate forms was much higher in the first two countries. Information of this nature could be utilized for obtaining first approximations to prevalence rates by limiting the examination of patients to a minimum, without attempting to classify them as $\mathrm{MB}$ or $\mathrm{PB}$, in the first instance.

Table 3

\begin{tabular}{lc}
\hline Country & $\begin{array}{c}\text { Prevalence rates } \\
\text { (Male: Female) }\end{array}$ \\
\hline Cameroon $^{4}$ & $1 \cdot 3$ \\
Thailand $^{4}$ & $1 \cdot 3$ \\
Nigeria $^{4}$ & $0 \cdot 84$ \\
S. India (Tirukoilur) & 5 \\
Myanmar (Regd. 1989) $^{6}$ & $1 \cdot 5$ \\
\hline
\end{tabular}


Again the sex ratio of patients in any society seems to be relatively constant, males having in general a higher prevalence rate. Table 3 gives the sex ratios from a selected number of countries.

Although, in population surveys, by house-to-house visits both males and females can be examined, there are some special problems with regard to each sex. For example, to examine females completely, one may need to recruit female workers. In sample surveys it is important to cover the population selected. At least a sample of the nonrespondents in the first round have to be traced to see how their nonresponse is related to the presence of the disease. This tracing of nonrespondents is usually time-consuming and needs a lot of effort. A knowledge of the approximate sex ratio of cases could facilitate limiting the tracing of nonrespondents to one of the sexes, according to the circumstances. For example, if the nonresponse from males is essentially due to their being away at work, complete coverage can be sought for women and the rates for males extrapolated. On the other hand if there are practical difficulties in examining women, it may be better to extrapolate from the male prevalence rates. It is understood that for practical reasons, in general, both males and females will be examined. The suggestions for extrapolation are applicable only at the analysis stage, when for some reason there are reasons to conclude that survey of one of the sexes is not perfect.

\section{(ii) Extrapolation from registered cases}

Practically all countries report the number of cases on their registers. In many situations there are comments by leprologists that the true number of cases could be so many times

Table 4. Kohn Kaen, Thailand, ${ }^{8}$ sample survey, 1962

\begin{tabular}{lccccc}
\hline Sample & $\begin{array}{c}\text { Population } \\
\text { examined }\end{array}$ & $\begin{array}{c}\text { Registered } \\
\text { cases }\end{array}$ & All cases & Reg./All & Block (\%) \\
\hline 1 & 3019 & 25 & 34 & $73 \cdot 5$ & \\
2 & 2448 & 34 & 49 & $69 \cdot 4$ & \\
3 & 2457 & 36 & 56 & $64 \cdot 3$ & \\
$4+5$ & 3711 & 20 & 31 & $64 \cdot 5$ & \\
6 & 3291 & 4 & 5 & $80 \cdot 0$ & \\
$7+8+9$ & 3932 & 22 & 30 & $73 \cdot 3$ & All 18,858 \\
& 18,858 & 141 & 205 & $68 \cdot 8$ & \\
& & & & &
\end{tabular}

Table 5. Orissa, India, 1988- $89^{9}$

\begin{tabular}{lcc}
\hline District & $\begin{array}{c}\text { Total } \\
\text { cases }\end{array}$ & $\begin{array}{c}\text { Voluntarily } \\
\text { reporting (\%) }\end{array}$ \\
\hline Ganjam & 4090 & $47 \cdot 3$ \\
Puri & 3602 & $43 \cdot 7$ \\
Cuttack & 4998 & $57 \cdot 9$ \\
\hline
\end{tabular}


those on the registers. For example, in $1987 \mathrm{Chitimba}^{7}$ reported for Nigeria that 'there are 282,000 registered patients out of a population of 100 million people and it is estimated that there are one million sufferers. An appraisal conducted in 1975 puts the prevalence rate of registered cases between $0 \cdot 1$ and 17 per thousand with a mean of five per thousand'. Thus the prevalence of registered cases was $2 \cdot 82$ per thousand and the national estimate is 5 per thousand. Tables 4 and 5 show examples from certain other areas of the world where there is a possibility of comparing the prevalence rates of registered cases with the true rates in the population.

What emerges from these comparisons is: (1) given a country's situation as regards leprosy services, the registered cases are a fairly constant proportion of all cases in that area, year by year and locality by locality (provided each locality is sufficiently large); (2) the proportion of $\mathrm{MB}$ cases or proportion of children can be quite different among the registered group when compared to all the cases. It is just that the total numbers seem to be well correlated irrespective of the composition of the two groups.

The merit of this approach is that it is least expensive. In many countries, limited surveys in selected areas already provide the type of relationship that exists between registered cases and all cases, and this relationship can be used to provide an extrapolating factor to derive the true prevalence from known registered cases. Undoubtedly, this would be a first approximation but may be adequate for planning purposes.

Issues involved in this approach are:

(1) there should be a leprosy service in the area with reasonable registration procedures;

(2) there should be a means to estimate the extent of multiple registration in different centres or services;

(3) a distinction should be made between areas or centres where the case-reporting is voluntary or passive and areas where there is an active case-finding programme. The extrapolation factors could be vastly different in the two types of area; and

(4) a distinction may have to be made between the population living within accessible distance of a leprosy service and others.

In any case pilot studies on a small scale should be carried out to derive an extrapolating factor or confirm the validity of one already being used.

\section{(iii) Extrapolation from child prevalence}

In many areas of the world, especially in hyperendemic areas under dapsone monotherapy, the prevalence rates of leprosy among children, 5-14 years of age, were closely correlated to the overall prevalence rates as Table 6 illustrates. ${ }^{8}$

Table 6

\begin{tabular}{lc}
\hline Country & Child prevalence rate/Total prevalence rate \\
\hline Myanmar (69-72) & $0 \cdot 79$ \\
Shwebo (Myanmar) (63) & $1 \cdot 04$ \\
N. Nigeria (60) & $1 \cdot 14$ \\
India (62-67) & $0 \cdot 92$ \\
\hline
\end{tabular}


The above relationship suggests that the total prevalence rates can be extrapolated from child prevalence rates if the latter can be ascertained. It should be noted that the proportions of $\mathrm{MB}$ and $\mathrm{PB}$ cases could be different for the two groups although the overall rates for all types may be closely related as above. Dr E Declercq ${ }^{8}$ has pointed out that 'there seems to be a strong correlation between the prevalence rate of leprosy for children and the total prevalence rate if studied in different areas of the same country and that the rates remain stable over the years within one area. However this relationship is not similar in different countries'.

If not already known from past experience based on prevalence surveys, etc. this should first be established or ascertained from pilot investigations. The advantage of extrapolating from child prevalence rates is that it is relatively quicker and less expensive to examine a large number of children, e.g. school surveys.

As an illustration of the savings involved, suppose it is known that the overall prevalence rate is about 1.25 times that of the child prevalence rate. If by examining 30,000 children we obtain a rate of 5 per 1000 we might be able to state that the overall rate is $6 \cdot 3$ per thousand with a $95 \%$ confidence interval between $5 \cdot 3$ and $7 \cdot 3$. To make a similar statement by actual population surveys, with cluster sampling, we might need a sample size of 50,000 . Of course the 30,000 children may be drawn from a population of 150,000 , but house-to-house visits are avoided and large numbers are examined at selected places such as schools. Further it is easier to examine children completely and nonresponse rates can be reduced considerably.

The issues involved in this approach are:

(1) it is important to know the proportion of children that attend school and the social strata from which they are drawn;

(2) one should make sure that the children attending school do not represent a 'selected' segment of the population;

(3) in the above case other possibilities such as co-operation with the immunization services, should be explored so as to obtain a good representation; and

(4) the school-starting age could vary from country to country. A choice should be made of the most appropriate age-group to be studied. Perhaps for operational reasons all children in the school should be examined recording the ages at the same time. The splitting by appropriate age-group could be done at the analysis stage.

\section{(iv) Rapid village surveys}

In this procedure an intensive effort is made to educate the population of the villages on symptoms of leprosy and the efficacy of modern drugs. The team visits the villages after this preparatory phase and with the help of key local personnel, individuals with suspected symptoms are asked to present themselves in a central place for examination. The method is easy to carry out as it does not require house-to-house visits, and the efficiency of the procedure should be estimated by comparing it with the traditional type of survey, at least in a few settings.

Any of the abovemethods, preferably a combination of them, could provide estimates of prevalence with a degree of precision acceptable for planning purposes. 


\section{SITUATIONS WHERE IT IS 5 YEARS OR MORE SINCE THE INTRODUCTION OF MDT}

In this situation, from the experience obtained so far, it is seen that there is a drastic reduction in the prevalence of registered cases, while the numbers of newly detected cases year by year continues undiminished at least for a number of years. Sometimes, voluntary reporting of hitherto undetected cases is prompted by the knowledge of the possibility of cure with MDT and an upsurge in 'newly detected' cases is seen during the first few years after MDT is introduced. In this situation the epidemiological pattern of the disease such as the proportion of $\mathrm{MB}$ cases, proportion of child prevalence and even ratio of registered cases to all cases, can change from year to year and the rapid methods suggested above may not be applicable.

The following procedure is suggested in such situations. It is assumed that because of treatment by MDT there is a drastic reduction in transmission of the disease at least from those treated. The new cases that are reported are presumably drawn: (1) from a pool of hitherto undetected cases coming now, of ten voluntarily, for treatment; (2) from cases infected prior to MDT but developing manifest disease only now; and (3) cases infected and developing the disease after the introduction of MDT. Data from two typical situations are presented in Table 7 to illustrate the dramatic reduction in prevalence rates

Table 7

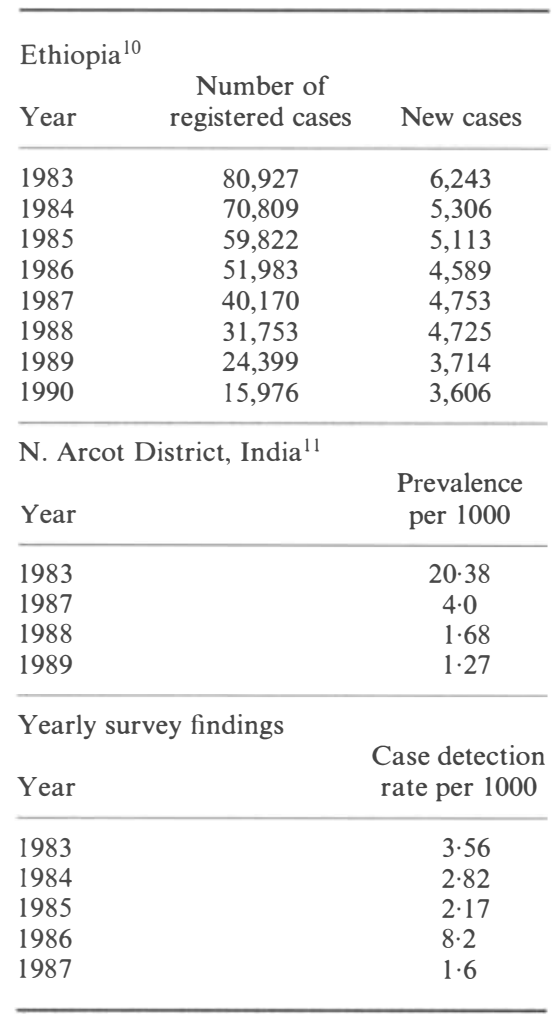


after MDT and the relatively slower rate of reduction in the annual new-case detection rate.

Thus in areas where there has been a vigorous implementation of MDT the prevalence over a period of 5 years drops dramatically. From the North Arcot experience it would appear that a good many of the cases that remained undetected (and unregistered) earlier have reported for treatment. Thus the numbers on the registers at the end of 5 years of MDT should be close to the actual number of cases in the community. On making enquiries from the leprosy workers in Tamil Nadu, the missing cases should at the most be $30 \%$ of those on the register. Thus the prevalence rate in N. Arcot at the end of 1989 should not be more than 1.7 per thousand-information that should be adequate for future planning of the leprosy programme.

In Ethiopia it has always been assumed that the registered cases as at 1985 represented half the number of true cases. In 1985 there were 59,822 cases on the register and this has been reduced to 15,976 cases in 1990 . If it is assumed that there is no reduction in the cases not on the register in 1985, an upper limit to the caseload in 1990 would be 75,798 or 1.5 per 1000 .

Thus in areas with MDT for 5 years or more the cases on register plus a small percentage should provide as good an estimate as any that can be obtained. The small 'correction factor' can usually be provided by the leprosy workers in that area and could well serve as an upper limit. At the expected rate of prevalence any imaginable sample survey could not provide estimates more precise.

\section{AREA WITH MDT OF LESS THAN 5 YEARS}

For estimation purposes this is perhaps the most difficult situation. The rate of reduction

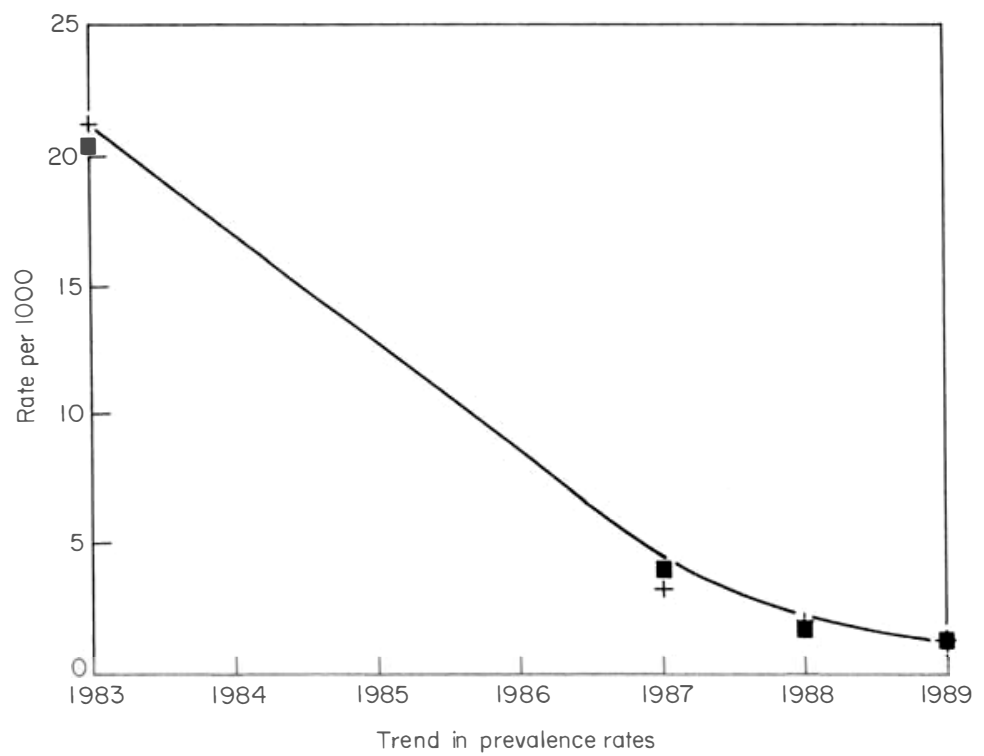

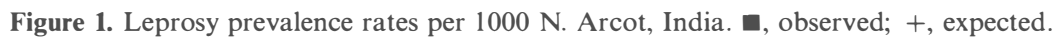


Table 8

\begin{tabular}{lc}
\hline $\begin{array}{l}\text { No. of years } \\
\text { after MDT }\end{array}$ & $\begin{array}{c}\text { Prevalence as a \% of } \\
\text { prevalence before MDT }\end{array}$ \\
\hline 0 & 100 \\
1 & 62 \\
2 & 39 \\
3 & 24 \\
\hline
\end{tabular}

in prevalence is rapid and steep. The rate of reduction would be substantial even during the period of a sample survey so that the result of the survey would no longer represent a 'point-prevalence'. The epidemiological pattern of the disease changes drastically from year to year. For example, because of the longer duration of treatment for MB cases the proportion of such cases still on the registers would appear to be increasing. Thus the rapid methods suggested above may not be satisfactory. On the other hand one can draw from the experience from areas where MDT has been introduced for more than 5 years. Figure 1 shows the exponential regression curve fitted to the available data from N. Arcot in India. Interpolating from this curve the trend in the prevalence rates would seem to be as in Table 8.

Experience with MDT is fast growing in many areas of the world. The above type of statistical analysis based on larger databases and in different country situations could improve the accuracy of the interpolations.

\section{References}

WHO Expert Committee on Leprosy, Sixth report. Tech. Report Series 768 (1988).

2 Bechelli LM, Matinez Dominguez V. The leprosy problem in the world. Bull. WHO, 1966; 34.

3 Sundaresan TK, Sansarricq H. \& Noordeen SK. Sample Surveys in Leprosy-An Introductory Manual (WHO/CDS/LEP/86.1).

4 Bechelli LM, Martinez Dominguez V, Patwary KM. WHO epidemiological random sample surveys of leprosy in Northern Nigeria (Katsina), Cameroon and Thailand (Khon Kaen). Int J Lepr, 1966; 34, No. 3.

5 Radhakrishna S, Christian M, Nair NGK. A 20-year study of the leprosy control programme at the Government Leprosy Treatment and Study Centre at Tirukoilur in South India. Ind J Med Res, 1982; 76: 1835.

6 Leprosy Control Program-Annual Report 1989. (Myanmar)-Leprosy Control, Dep. of Pub. Health (June, 1990).

7 Chitimba NM. Combined Leprosy/Tuberculosis Programme through Primary Health Care in Nigeria. Assignment Rep. 5 December 1986-4 November 1987.

8 Report of a meeting on methods for the Rapid Assessment of the Leprosy Situation-Geneva, 15-16 April 1988 (WHO/CDS/LEP/88.2).

9 Sundaresan TK. Estimation of leprosy prevalence in India and validation of district-wise prevalence data. Temp. Adviser report (25 Aug 90-5 Oct. 90).

10 Annual Report of National Leprosy Control Programme (Ethiopia). 1989-1990. Ministry of Health, People's Democratic Republic of Ethiopia.

11 Ekambaram V, Rao MK. Changing picture of leprosy in North Arcot District, Tamil Nadu after MDT. Ind J Lepr, 1989; 61, No. 1 . 